\title{
TRABAJO Y CUESTIÓN SOCIAL DESDE LA PERSPECTIVA DE EDLENE PIMENTEL ${ }^{1}$
}

\section{WORK AND SOCIAL ISSUE FROM THE EDLENE PIMENTEL'S PERSPECTIVE}

\section{Sonia Angulo Brenes*}

\section{RESUMEN}

El objetivo del artículo es contribuir al análisis de la cuestión social desde el trabajo como fundamento ontológico de la sociedad, a partir de la contribución teórica y analítica de Edlene Pimentel, con el fin de comprender la sociedad contemporánea y especialmente, las "manifestaciones de la cuestión social". A partir de ahí, discutir las posibilidades y los límites de las lecturas de Robert Castel y Pierre Rosanvallon sobre la cuestión social y principalmente, sobre sus "nuevas" manifestaciones en una sociedad donde la contradicción capital/trabajo no ha desaparecido.

PALABRAS CLAVE: SOCIEDAD CONTEMPORÁNEA * SALARIO * ESTADO * DESEMPLEO

\section{ABSTRACT}

The article's objective is to contribute to the analysis of the social issue in the light of work as society's ontological basis, regarding the theoretical and analytical contribution of Edlene Pimentel in order to understand the contemporary society and mainly the "social issues manifestations". Hence, from there, discuss the possibilities and limitations of Robert Castel and Pierre Rosanvallon's interpretations about the social issue and, specially, its "new" manifestations in a society where the capital/work contradiction has not yet disappeared.

KEYWORDS: CONTEMPORARY SOCIETY * WAGES * STATE * UNEMPLOYMENT

1 Este artículo es producto del proyecto de investigación titulado "Cuestión Social, fundamentos del Trabajo Social y tradición marxista en la Escuela de Trabajo Social de la Universidad de Costa Rica 2004-2016" de la Escuela de Trabajo Social de la Universidad de Costa Rica. 


\section{INTRODUCCIÓN}

En la discusión actual de las ciencias sociales para la comprensión de la sociedad contemporánea, una de las categorías fundamentales es la cuestión social, la cual ha sido estudiada por diferentes autores $y$ autoras, entre ellos, los dos más importantes han sido los franceses Robert Castel y Pierre Rosanvallon. En América Latina se pueden identificar estudios tanto en Argentina como Brasil, los cuales proponen discusiones históricas y teóricas sobre esta categoría. En este sentido, interesa resaltar tres autores, por un lado, Ricardo González Leandri con su análisis de lo que llama la "temprana cuestión social" en Argentina $y$, por otro lado, desde la base marxista de análisis se encuentran José Paulo Netto y Alejandra Pastorini, quienes analizan la cuestión social y sus manifestaciones desde el surgimiento de la sociedad capitalista.

A partir de las lecturas citadas es que surge la necesidad de buscar otras contribuciones sobre este tema, así el objetivo del artículo es contribuir al análisis de la cuestión social desde el trabajo como fundamento ontológico de la sociedad, a partir de la contribución teórica de Edlene Pimentel, quién discute sobre las bases del capitalismo y las formas en que se expresan las diferentes configuraciones, tales como, la pobreza y el desempleo en la llamada crisis estructural del capital. En este sentido, la base del artículo es analizar su propuesta teórica a través de su texto Uma "nova questão social"? Raizes materiais e humano-sociais do pauperismo de ontem e de hoje ${ }^{2}$, el cual es producto de su tesis doctoral realizada en el año 2005, en donde, a partir de la base teórica de Karl Marx e István Mészáros, realiza un acercamiento a la categoría y se confronta con los dos autores franceses citados.

La lógica del artículo se establece desde dos líneas, por un lado, la síntesis de los postulados de Castel y Rosanvallon sobre la categoría analizada $y$, por otro lado, la identificación de las contribuciones de la autora para debatir

2 Traducción en español “¿Una "nueva cuestión social"? Raíces materiales y humano-sociales de la pobreza de ayer y de hoy". sobre la cuestión social y sus manifestaciones, especialmente, la pobreza y el desempleo. En este sentido, la propuesta no pretende establecer una comparación entre las tres posturas sino solamente exponer los aspectos más importantes de cada una y las críticas desde Edlene Pimentel a los dos autores franceses citados, asimismo, mostrar los aportes de la propuesta escogida, con la finalidad de discutir las posibilidades y límites de las lecturas sobre la cuestión social y especialmente, sobre las "nuevas" manifestaciones en una sociedad donde la contradicción capital/trabajo no ha desaparecido.

\section{CUESTIÓN SOCIAL Y SUS DEBATES}

Los aportes de Robert Castel y Pierre Rosanvallon para el estudio y comprensión de la cuestión social fueron fundamentales para entender la situación del salario y el papel del Estado a lo largo del siglo xx, sin embargo, sus análisis se limitaron a una propuesta reformista ante la crisis de la década de 1970. Asimismo, es necesario indicar que el debate sobre la cuestión social remite a tres aspectos sobre su surgimiento histórico, su comprensión teórica $y$ la discusión sobre la existencia de una nueva cuestión social en la sociedad contemporánea.

En el caso de Robert Castel su propuesta se centra en el salario como base $y$ punto de partida para su análisis, y establece una distinción entre "problemática del socorro" y "problemática del trabajo". Su análisis se centra en la relación del asalariado en la sociedad contemporánea:

(...) es la cuestión del salariado lo que de este modo se plantea, es decir, la necesidad creciente de recurrir a la salarización, y al mismo tiempo la imposibilidad de regular una condición salarial, por la persistencia de tutelas tradicionales que encorsetan el trabajo en las redes rígidas de obligaciones sociales, y no económicas (Castel, 1997, p. 31).

Su base analítica remite a la relación entre asalariados y no asalariados, estos últimos desligados de toda relación contractual $y$ de posibilidad de satisfacer sus necesidades. 
En términos generales, su análisis se centra en esta contradicción, así define a la cuestión social como una "aporía fundamental en la cual una sociedad experimenta el enigma de su cohesión y trata de conjugar el riesgo de su fractura" (Castel, 1997, p.20).

Desde una postura reformista su solución consiste en "hacer lo social" y esto significa "trabajar sobre la miseria del mundo capitalista", es decir, sobre los efectos perversos del desarrollo económico para promover una integración de esas poblaciones más desposeídas. En este sentido, para el autor la "cuestión social" no se restringe tan solo a la pobreza ni a la miseria, sino se centra en los desequilibrios $y$ perturbaciones que se generan en la relación salarial. A partir de este elemento, el autor (1997) determina cinco circunstancias que permiten explicar cronológicamente las condiciones de esta relación a partir de los inicios de la industrialización hasta el fordismo:

1) Una división de esa relación entre quienes poseen trabajo regular y permanente y los "excluidos" del mercado del trabajo.

2) La fijación del trabajo a la regulación de una conducta obrera con el taylorismo.

3) El acceso del obrero al consumo a través de su relación salarial.

4) El acceso del obrero a la propiedad social y a los servicios públicos que brinda el Estado.

5) El reconocimiento del trabajador como miembro de un colectivo fundamentado en un estatuto social.

Estas transformaciones impactaron en la relación salarial y conllevaron a un cambio en las condiciones obreras $y$, en general, de la sociedad, de ahí su vínculo con el análisis de la metamorfosis de la cuestión social. A partir de esta, remite a una serie de situaciones que están sucediendo en la sociedad contemporánea, tales como, el desempleo masivo, la precarización, la crisis del Estado Social y las condiciones de lo que llama los "supernumerarios", es decir aquellas personas excluidas de la relación salarial.
El surgimiento de la cuestión social remite a 1830 "(..) a partir de la toma de conciencia de las condiciones de vida de poblaciones que eran a la vez agentes $y$ víctimas de la revolución industrial. Era la cuestión del pauperismo" (Castel, 1997, p.20). Sin embargo, aunque indica que ese es el punto de partida del fenómeno, explícitamente se podría hablar de un surgimiento implícito antes del siglo XIX pero que se extiende hasta mediados del siglo $x x$. Por lo que se recorre un camino desde el siglo XIV (1349) en la sociedad feudal hasta la organización social por condiciones salariales, en esta última "la "cuestión social" consistía (...) precisamente en la toma de conciencia de que esa fractura central puesta en escena a través de las descripciones del pauperismo podía llevar a la disociación del conjunto de la sociedad" (Castel, 1997, p. 325).

Este recorrido histórico lo determina a partir de tres categorías: "condición proletaria" (el proletario es una parte del proceso de industrialización y este depende de ello para su reproducción), la "condición operaria" (que implica una nueva relación salarial que permite al obrero asegurar sus derechos y participar en la vida social) y la "condición salarial" (subordinación del operario al salario).

La cuestión social se expresa en las personas "excluidas" de la sociedad, esta "(...) se plantea explícitamente en los márgenes de la vida social, pero "pone en cuestión" al conjunto de la sociedad" (Castel, 1997, p. 23). Esta exclusión de la relación salarial genera en los años 60 , una precarización del trabajo que alimenta la vulnerabilidad social y produce el desempleo y la desafiliación, de ahí que el autor (1997) discute la configuración de una "nueva cuestión social" que tiene como eje central la precarización social desencadenada por el proceso global de reestructuración capitalista, que resulta en la desestabilización de los Estados llevando a la vulnerabilidad de las masas.

Su solución ante esto es el regreso a un Estado Social que permita armonizar los conflictos para mantener la cohesión social y que contribuya a la configuración de una sociedad donde el trabajo no se constituya en el eje fundamental, fomentando políticas de inserción, 
que "(...) obedecen a una lógica de discriminación positiva: se focalizan en poblaciones particulares y zonas singulares del espacio social, y despliegan estrategias específicas" (Castel, 1997, p. 422).

Ante este panorama que presenta el autor, la pregunta que surge es ¿cuáles son las contribuciones y críticas a su propuesta?, como indica Pastorini (2010) uno de los aportes más interesantes es el recorrido histórico por la relación salarial y sus múltiples particulares, denotando sus transformaciones.

Sin embargo, las críticas refieren a varios elementos, el primero a la lectura reformista que propone de la cuestión social y de la sociedad, en donde, la solución remite a la posibilidad de cohesión social en un mundo, que como el autor expone, es cada vez más contradictorio $y$ excluyente, $y$ por lo tanto, el cuestionamiento que surge es ipor qué hablar de una nueva cuestión social si las condiciones históricas de desigualdad social se siguen manteniendo $y$ más bien cada vez se recrudecen?

Finalmente, de la propuesta de Robert Castel cabe preguntarse si es posible pensar en el regreso de un Estado Social frente a las propuestas conservadoras que se imponen con más fuerza en diferentes países europeos y americanos, en tanto, las políticas de inserción presentan la misma contradicción, incluyendo a unos mientras excluye a otros.

Otra mirada interesante es la de Pierre Rosanvallon, el cual fundamenta su análisis a partir del desarrollo del Estado de Providencia francés $y$ se basa en una forma de solidaridad determinada por el contrato social. Este se basa en una relación entre el riesgo y el seguro, por tanto, "no es como la asistencia de un socorro consentido; él representa la ejecución de un contrato en el que el Estado y los ciudadanos están igualmente implicados" (1995, p.24).

A partir de los años 80, el crecimiento del desempleo y el congelamiento de los salarios producen la reducción del número de los contribuyentes, lo que implica que el gobierno reduzca gastos para estimular a las empresas y crear empleos, lo que dificulta la atención a los desempleados y por esto es que su propuesta es una gestión social del desempleo.
Para Rosanvallon (1995), la cuestión social es una expansión del pauperismo que se identifica con la propia industrialización. Su surgimiento remite a fines del siglo XIX debido a los efectos de la sociedad industrial que con el paso del tiempo, a partir del siglo xx, se agudiza $y$ se transforma en una nueva cuestión social:

El advenimiento de una nueva cuestión social se traduce en una inadaptación de los viejos métodos de gestión de lo social. Es testimonio de ello el hecho de que la crisis del Estado providencia, diagnosticada desde fines de los años setenta, haya cambiado de naturaleza (Rosanvallon, 1995, pp. 7-8).

En este sentido, el autor expone una relación entre esta nueva cuestión social y una crisis filosófica que empieza a surgir a partir de la crisis de los años 70, esa crisis se manifiesta en “(...) dos problemas mayores: la desintegración de los principios organizadores de la solidaridad y el fracaso de la concepción tradicional de los derechos sociales para ofrecer un marco satisfactorio en el cual pensar la situación de los excluidos" (1995, p.10).

Por esto su propuesta de solución ante las situaciones que se presentan en la sociedad contemporánea se basa en tres principios: refundar la solidaridad, renacer la nación y redefinir los derechos para fortalecer el Estado providencia. Para que se cumpla con esta finalidad se debe redefinir los valores, especialmente, la comprensión de la igualdad y generar un "reformismo del individuo" “(...) adaptado a la pluralidad de las situaciones en las que se encuentran los actores, apuntando a dotarlos de medios adecuados de acción y defensa" (Rosanvallón, 1995, p. 214). Finalmente, la redefinición del progreso social y de una reconstrucción política que logre la consecución de un Estado providencia activo.

A partir de esta breve síntesis, tres críticas que realizan diferentes autoras a la propuesta de Rosanvallón refieren a lo siguiente:

$\diamond \quad$ Pastorini (2010) cuestiona su comprensión, en tanto, indica que Rosanvallón sin cuestionar la sociedad capitalista, 
responde a la solución de las problemáticas sociales con el fortalecimiento de un Estado providencia que, a través de una nueva ciudadanía, respondería de manera superficial a estas manifestaciones de la cuestión social.

$\diamond \quad$ La lectura de una nueva cuestión social que excluye cada vez es contradictoria con su solución hacia la solidaridad, ya que está determinada por la contribución de los individuos, volviendo otra vez la mirada en quienes se encuentran integrados y no frente a los excluidos (Pimentel, 2012).

$\diamond \quad$ Según Pimentel (2012), la limitación más profunda se fundamenta en que su propuesta se basa en combatir la pobreza sin tocar las bases de la sociedad capitalista y sus contradicciones, de ahí su propuesta a la vuelta a un Estado Social que remedie los "problemas sociales" para la regulación del mismo sistema.

Finalmente, frente a estas dos posturas que han sido fundamentales para la comprensión de la cuestión social y de su reproducción histórica en la sociedad capitalista, es que se presenta la propuesta de Edlene Pimentel, ante la búsqueda de análisis críticos que permitan profundizar en esta categoría.

\section{CONTRIBUCIONES Y APORTES DE LA PERSPECTIVA DE EDLENE PIMENTEL}

Pimentel (2012) presenta lo que llama un esbozo de una interpretación crítica de la cuestión social, la cual contiene varios aportes interesantes que se presentan a continuación:

1) La comprensión de la cuestión social desde sus determinaciones esenciales $y$ de sus expresiones como trazos indisolubles del modo de ser de la sociedad capitalista a partir de las formulaciones de Marx y Mészáros.

Esta autora se posiciona frente a la discusión de la "nueva cuestión social" y de una reformulación del Estado Social que logre la resolución de las expresiones de la cuestión social como la precarización del trabajo o la exclusión social. Las razones que determinan este posicionamiento son dos, por un lado, no puede existir históricamente una "nueva" cuestión social, en tanto, la contradicción capital/trabajo sigue siendo fundamental para la reproducción de la sociedad contemporánea, y por otro lado, la reconstitución de un Estado Social que solucione aquellas manifestaciones estructurales propias de la sociedad capitalista, se convertiría en una preocupación por preservar el orden capitalista que las produce más que su superación.

Retomando a Mészáros, su postura es "ir más allá del capital", superar la contradicción capital/trabajo, por ende, la cuestión social frente a las salidas brindadas por Castel y Rosanvallon de una propuesta de una nueva redefinición del Estado Social, la cual, se ve imposibilitada debido a la naturaleza estructural de la crisis a partir de los años 70 .

2) El abordaje de la emergencia del fenómeno del pauperismo en el siglo XIX desde la Ley General de la Acumulación Capitalista propuesta por Marx (2010), en donde, su esencia se encuentra en el proceso de acumulación capitalista que, de forma antagónica, genera al mismo tiempo la acumulación de riqueza por parte del capitalista frente a la miseria y pauperización del trabajador:

Ese proceso de acumulación se da porque la fuerza de trabajo comprada por el capitalista no es destinada para satisfacer sus necesidades individuales. Aunque el trabajador reciba un salario por la venta de su fuerza de trabajo, que responda a tales necesidades con vistas a aumentar el consumo de alimentos, vestimenta, etc., aun así el grado de explotación a que está sometido impide que él se libere de su condición de dependencia y menos aún de la de trabajador asalariado (Pimentel, 2012, p. 141) $)^{3}$.

En esta constante contradicción, la apropiación del trabajo que genera producción de mercancías será concentrada por la clase capitalista y así se garantiza la reproducción del

\footnotetext{
3 Traducción propia.
} 
capital. Su postura se constituye en superar la comprensión superficial de la cuestión social, en la cual, la centralidad se presenta en los aspectos fenoménicos como el pauperismo, sin penetrar en la aprehensión de la esencia del problema $y$ de sus determinaciones, ya que es el propio proceso de acumulación capitalista que multiplica constantemente la miseria de la masa de trabajadores asalariados.

En este sentido, es crítica con el posicionamiento de Castel y Rosanvallon, en tanto, su comprensión de la cuestión social se presenta como "(...) una forma articulada entre el problema de pauperización de los trabajadores y la cuestión política manifiesta en su reacción a las precarias condiciones de vida $y$ de trabajo de la sociedad industrial reciente"4 (Pimentel, 2012, p.139). Desde su análisis, la postura de estos dos autores se fundamenta en una perspectiva conservadora, pues lo que intentan es alertar o prevenir los riesgos de la insurrección obrera y así preservar el orden socioeconómico imperante.

3) La superación de la comprensión de la cuestión social como pauperismo, pues la autora expone que el surgimiento de esta categoría se diferencia con el avance de la pobreza. De tal forma que su esencia se encuentra siguiendo a Marx en el proceso de acumulación capitalista que, al mismo tiempo que genera la acumulación, se propaga la miseria, siendo esta última expresión de la cuestión social:

Por tanto, aprender ese fenómeno en su inmediatez enfatizando apenas en su aspecto político, sin remitir a su sistema de causalidades, localizado en la base económica del modo de producción capitalista no va de modo alguno, revelar $y$ equiparar verdaderamente la cuestión (Pimentel, 2012, p.142).

De tal manera que desde este punto analítico y comprensivo de la sociedad, las soluciones expuestas por los autores Castel y Rosanvallon se convierten en mecanismos que minimizan las manifestaciones de la cuestión social a través de paliativos desde la reformulación de un Estado Social.

4) Una crítica a la lectura de la existencia de una nueva cuestión social que surge de la agudización del desempleo crónico y de la pobreza. Pimentel frente a esta comprensión remite a István Mészáros para plantear la condición del desempleo crónico no solo en los países del Tercer Mundo o periferia, sino también en los países de economía central y discute que el fenómeno del desempleo crónico en la sociedad capitalista contemporánea hace emerger "(...) las contradicciones y los antagonismos del sistema global en la forma potencialmente más explosiva"5 (Mészáros, 2006, pp. 224-225). Es por esta razón que todas las medidas creadas en el sentido de remediar el defecto estructural del desempleo creciente no aliviarán el problema, solo lo agravarán.

En primer lugar, un desempleo crónico que está en todos los ramos de la actividad, disfrazado de "prácticas de trabajo flexibles" para generar la política de fragmentación y de precarización de la fuerza de trabajo y mayor explotación en tiempo parcial. En segundo lugar, una reducción del patrón de vida de la población trabajadora con leyes y cambios cada vez más agresivas en sus condiciones:

Mészáros comenta la falacia que es la mistificación ideológica del discurso socialdemócrata de que el permanente dominio del capital sobre el trabajo puede llevar a la única forma viable de reproducción socioeconómica: la idealizada "sociedad de mercado" y las "oportunidades iguales" que pretendidamente una sociedad de esa naturaleza podría proporcionar a todos los individuos ${ }^{6}$ (Pimentel, 2012, p. 147).

La lectura de Castel y Rosanvallon se basan en el surgimiento de una "nueva cuestión social" en la sociedad contemporánea, para el primer autor surge a partir de la crisis en

\footnotetext{
$5 \quad$ Traducción propia.
}

6 Traducción propia. 
la década de los años 70 en la que la cuestión social se transforma $y$ su eje se convierte en la precarización del trabajo y el desempleo. Para el segundo autor, expone que es en los años 80, en donde aparecen nuevas formas de precarización, desempleo y nuevas formas de pobreza y se basa en la "desestabilización de la masa trabajadora" como elemento que confirma expresiones nuevas. Ante esto, las soluciones brindadas por estos autores se convierten en respuestas reformistas que no permitirán superar los antagonismos y expresiones de desigualdad que se recrudecen en la sociedad capitalista contemporánea.

La autora (2012) se posiciona sobre estas lecturas precisando tres elementos fundamentales sobre el trabajo, la precarización y el desempleo:

$\diamond \quad$ La comprensión del desempleo crónico que se encuentra en todas las actividades del empleo y que se disfraza de "prácticas de trabajo flexibles" que conllevan la fragmentación y la precarización de la fuerza de trabajo.

$\diamond \quad$ Una reducción del patrón de vida de la clase trabajadora y su poder adquisitivo, así como, una mayor explotación de la masa de trabajadores de tiempo parcial.

$\diamond \quad$ La constitución de un discurso democrático que se fundamenta en leyes cada vez más autoritarias y que se combina con actitudes más agresivas del capital contra su fuerza de trabajo.

En este sentido, la situación del desempleo, tanto de los países centrales como periféricos, se agudiza cada vez más:

El desempleo crónico, que golpea también a los países del capitalismo avanzado, inclusive las clase medias, van generando mucho sufrimiento no solamente para los trabajadores no calificados, sino también para aquellos cualificados que disputan las pocas vacantes existentes en el mercado, con un ejército enorme de desempleados (Pimentel, 2012, p. 159). ${ }^{7}$
Por tanto, la lectura de una existencia de una nueva cuestión social que se presenta por supuestas nuevas formas de pobreza, de precariedad, de desempleo en masa, de exclusión, conforme postulan Castel y Rosavanllon, en realidad son cambios en las manifestaciones de la cuestión social ocasionados por la crisis estructural del capital en los años 70, resultante de su proceso de expansión y acumulación que desencadena la deshumanización y destrucción global de la sociedad capitalista y del género humano. Su posicionamiento es que el avance de un desempleo estructural no reside en las transformaciones sufridas en la coyuntura del propio empleo (como indican los autores citados), sino en el interior de la propia estructura del capital.

5) Una lectura crítica sobre el regreso de un Estado Social o de Providencia, se discute y polemiza, pues Pimentel (2012) desde una lectura histórica explica como los autores al hablar de un posible fortalecimiento de este tipo de Estado, confunden la reproducción histórica de la crisis de 1929 y su solución de un Estado Social, con la crisis estructural del capital que aparece a partir de los años 70:

Todo esto porque el tendón material del capital es el trabajo y no el Estado. Por tanto, reestructurar el Estado en los términos propuestos no va a eliminar la precariedad del empleo, o desempleo en masa, la desafiliación, la exclusión social, como sugieren Castel y Rosanvallon ${ }^{8}$ (Pimentel, 2012, p. 150).

Pimentel expone que lo que ellos llaman nueva cuestión social en realidad se constituye en una vieja forma reinventada para afrontar unas problemáticas viejas reconfiguradas por la crisis estructural del capital. De esta manera, la comprensión de la cuestión social:

(...) originalmente expresa en el empobrecimiento del trabajador tiene sus bases reales en la economía capitalista. Políticamente, pasa a ser reconocida

$8 \quad$ Traducción propia.

$7 \quad$ Traducción propia. 
como problema en la medida en que los individuos empobrecidos se organizan, ofreciendo resistencia a las malas condiciones de existencia resultantes de su condición de trabajadores ${ }^{9}$ (2012, p. 155).

Esta lectura de cuestión social permite comprender que históricamente surge a partir de una contradicción fundamental entre el capital y el trabajo, que sus manifestaciones solo desaparecerán en el momento que sea superada, frente a esto, las soluciones que se piensan a través del Estado son superficiales e inmediatas, tal cual indica Mallardi:

La realidad muestra además que la sociedad capitalista reactualiza sus crisis y la necesidad de avanzar en explicaciones que permitan comprenderlas es un elemento clave en el desarrollo de las ciencias sociales. La tendencia a retomar argumentaciones sin identificar su correlato con la realidad aparece en muchas ocasiones como la primera opción, sin embargo la continuidad de la desigualdad social exige una crítica a éstas (2013, p. 429).

Finalmente, estas lecturas conservadoras recaen en lecturas que colocan al individuo como responsable de esta superación, siendo así que en la sociedad contemporánea la cuestión social sigue siendo la misma, en tanto su fundamento, la contradicción entre el capital $y$ el trabajo que posee en su esencia las raíces humano-históricas de la sociedad capitalista, no han desaparecido.

\section{ALGUNAS CONCLUSIONES}

La relevancia de la categoría cuestión social para la comprensión de la sociedad en que se vive, en tanto, histórica y teóricamente, permite entender la contradicción de la generación de acumulación frente a la miseria y como sus expresiones son constantemente sentidas por las clases sociales más empobrecidas, especialmente,

$9 \quad$ Traducción propia. la agudización de la pobreza, el desempleo crónico y la desigualdad social.

Asimismo, el texto brinda la posibilidad de generar debate entre los diferentes postulados teóricos que fundamentan una serie de lecturas sobre su comprensión, así como, surge la necesidad de buscar referentes críticos que permitan principalmente a las ciencias sociales, una comprensión y aprehensión de las configuraciones de la sociedad para lograr un acercamiento profundo a esas expresiones que se agudizan cada vez más producto de la crisis estructural del capital.

Los aportes desarrollados por la autora Edlene Pimentel sobre la cuestión social colocan el desafío de profundizar en el estudio de la categoría pero también de su reproducción en la sociedad en términos históricos. Asimismo, se busca cuestionar lecturas más reformistas que discutan el aparecimiento de una "nueva cuestión social" y de un Estado Social, que no logran resolver la magnitud de esas manifestaciones, en un contexto actual donde cada vez hay más conservadurismo y deshumanización.

Es relevante entender que desde esta postura no existe una nueva cuestión social sino más bien condiciones históricas producto de la crisis de finales de 1970, la cual generaró transformaciones en las manifestaciones de la cuestión social, tales como, el desempleo crónico, producto de la agudización de la contradicción capital/trabajo.

De ahí la importancia de que las ciencias sociales estudien $y$ analicen las condiciones de la sociedad contemporánea desde un posicionamiento crítico que permitirá el avance en su comprensión y de sus múltiples contradicciones.

\section{REFERENCIAS}

Castel, R. (1997). Las metamorfosis de la cuestión social. Una crónica del salariado. Buenos Aires, Argentina: Paidós.

González, R. (2010). La temprana Cuestión Social. La ciudad de Buenos Aires durante la segunda mitad del siglo XIX. Consejo Superior de Investigaciones Científicas: Madrid. 
Mallardi, M. (2013). La "cuestión social" mistificada: límites y tensiones en la "crisis" de la sociedad salarial. Cuadernos de Trabajo Social, 26(2), pp. 421-430.

Marx, C. (2010). El Capital. Crítica de la economía política. Tomo I. Libro 1. Proceso de producción del capital. Santiago, Chile: LOM Ediciones.

Mészáros, I. (2002). Para Além do Capital. Rumo a uma teoria da transição. São Paulo, Brasil: Editorial Boitempo.

Netto, J. (2003). Cinco notas a propósito de la "cuestión social". En: E. Borgianni, Y. Guerra y C. Montaño (orgs), Servicio Social Crítico: Hacia la construcción del nuevo proyecto ético-politico profesional (pp. 55-69). São Paulo, Brasil: Cortez Editora.
Pastorini, A. (2010). A categoria "Questão Social” em debate. São Paulo, Brasil: Cortez Editora.

Pimentel, E. (2012). Uma "nova questão social"? Raizes materiais e humanosociais do pauperismo de ontem $e$ de hoje. São Paulo, Brasil: Instituto Lukács.

Rosanvallon, P. (1995). La nueva cuestión social. Repensar el Estado providencia. Buenos Aires, Argentina: Ediciones Manantial.

Fecha de ingreso: 24/06/2019 Fecha de aprobación: 28/08/2019 
